\title{
Management of Unilateral Zygomaticomaxillary Complex Fracture - A Case Report
}

\author{
Vybhavi M. K. ${ }^{1}$, Prashanth V.2 ${ }^{2}$, Srinivas V. ${ }^{3}$ \\ 1, 2, 3 Department of ENT \& HNS, BGS Global Institute of Medical Sciences, Bengaluru, Karnataka, India.
}

\section{INTRODUCTION}

Zygomaticomaxillary complex (ZMC) fractures are relatively common. Zygomatic complex fractures with functional or aesthetic impairments often require surgical intervention. Treatment of ZMC fractures consists of reduction and fixation of the dislocated bone fragments to their original location.

The zygomaticomaxillary complex functions as a major buttress for the face and because of its prominent convex shape, is frequently involved in facial trauma. ${ }^{1} \mathrm{ZMC}$ fractures are also called tripod, tetrapod, quadripod, malar or trimalar fractures. They account for approximately $15 \%-23.5 \%$ of maxillofacial fractures. ${ }^{2,3}$ The aetiology of zygomatic complex fractures primarily includes road traffic accidents (RTA), violent assaults, falls and sports injuries. They are the second most common facial fracture after nasal bone fractures. ${ }^{3-6} \mathrm{ZMC}$ fractures are more common in men than women, and most commonly occur in the third decade of life. ${ }^{7-10}$ The main clinical features of zygomatic complex fractures include diplopia, enophthalmos, subconjunctival ecchymosis, extraocular muscle entrapment, cosmetic deformity, malocclusion and neurosensory disturbances of the infra-orbital nerve.10 The gold standard radiological investigation for evaluation of ZMC fractures is computed tomography (CT) scan. Surgical intervention is effective in cases of displaced and comminuted fractures involving functional and aesthetic defects, whereas a nonsurgical approach is often used for non-displaced fractures.11 Various surgical approaches and treatment strategies have been proposed to obtain a successful treatment outcome. Based on review of literature, it has been observed that the open reduction with internal fixation using mini plates and screws is the most commonly preferred treatment for displaced and comminuted fractures. ${ }^{10-12}$ Here, we report a clinical case of right zygomaticomaxillary complex fracture and its management.

\section{PRESENTATION OF CASE}

A 32-year-old female patient was presented to the out-patient department of Otorhinolaryngology with history of facial trauma following road traffic accident. She presented with complaints of pain over the right malar region of the face and restricted mouth opening (Fig. 1). There was no history of diplopia, disturbances in vision. No history of head injury, nausea, vomiting. On clinical examination, mild trismus, right side infraorbital paraesthesia and facial bruise over the right malar region was noted. Ophthalmologic consultation was done and globe injury was ruled out. There was no restriction of extraocular movements, no enophthalmos and vision was found to be normal.
Corresponding Author:

Dr. Vybhavi M. K.,

Department of ENT,

BGS GIMS, Kengeri - 560060,

Bengaluru, Karnataka, India.

E-mail: vybhavi999@gmail.com

DOI: $10.14260 / j e m d s / 2021 / 626$

How to Cite This Article:

Vybhavi MK, Prashanth $V$, Srinivas $V$. Management of unilateral zygomaticomaxillary complex fracture: a case report. J Evolution Med Dent Sci 2021;10(35):3070-3073, 10.14260/jemds/2021/626

Submission 05-05-2021, Peer Review 26-07-2021, Acceptance 02-08-2021, Published 30-08-2021.

Copyright (C) 2021 Vybhavi MK et al. This is an open access article distributed under Creative Commons Attribution License [Attribution 4.0 International (CC BY 4.0)] 
CT scan of maxillofacial bones was taken which showed fractures of the anterior and postero-lateral walls of the right maxillary sinus (Fig. 2, 3, 4). Fracture of the right inferior orbital margin, fracture with displacement of the right lesser wing of sphenoid and segmental fracture of the right zygomatic arch were also noted on CT scan. No evidence of any intracranial abnormalities was noted on CT scan. Similar findings were observed on 3D image reconstruction as well (Fig. 5, 6).

Patient was shifted to operation theatre after routine laboratory investigations and pre-anaesthetic check-up was done. Patient was intubated through an oro-endotracheal tube under general anaesthesia. Surgical access was through intraoral and transcutaneous infra-orbital approach (Figure 7, 8). Intra-orally, upper gingivobuccal sulcus incision was made from the canine to the first molar tooth. The mucoperiosteum was reflected to expose the fracture site in the zygomaticomaxillary buttress region. Infraorbital incision measuring $3 \mathrm{~cm}$ was given on the right side and soft tissue dissection was done to expose the inferior orbital margin. The infraorbital foramen along with the infraorbital nerve was identified and secured. Open reduction of the fracture in the zygomaticomaxillary buttress region and zygomatic arch fracture on the right side was done through the gingivobuccal sulcus incision via intra-oral approach. Fracture reduction was stable and showed adequate anatomic alignment. The fracture site in the right inferior orbital margin was exposed (Figure 9) and rigid internal fixation was done using titanium mini plate $1.5 \mathrm{~mm}$. Figure 10 shows adequate anatomic alignment and fixation with mini-plates at the infraorbital rim. The sulcus incision and the infraorbital incision was closed in layers using Vicryl 3 - 0 and Ethilon $4-0$ (Figure 11). Patient was treated with post-operative antibiotics and analgesics. The postoperative recovery was uneventful.

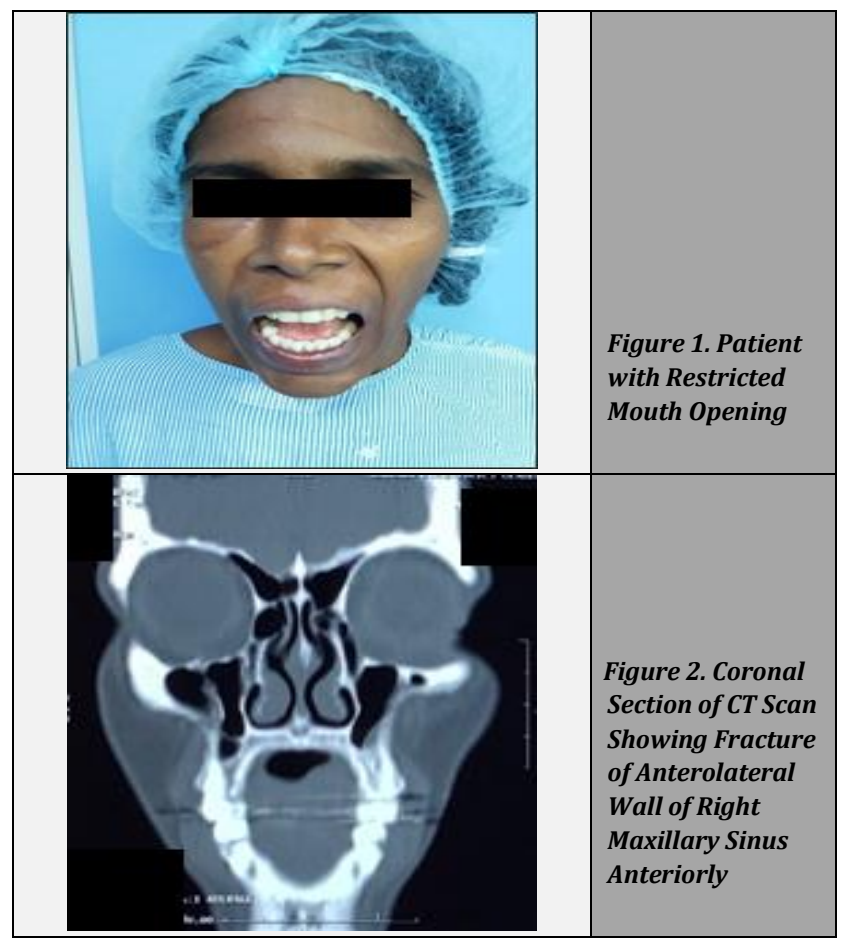

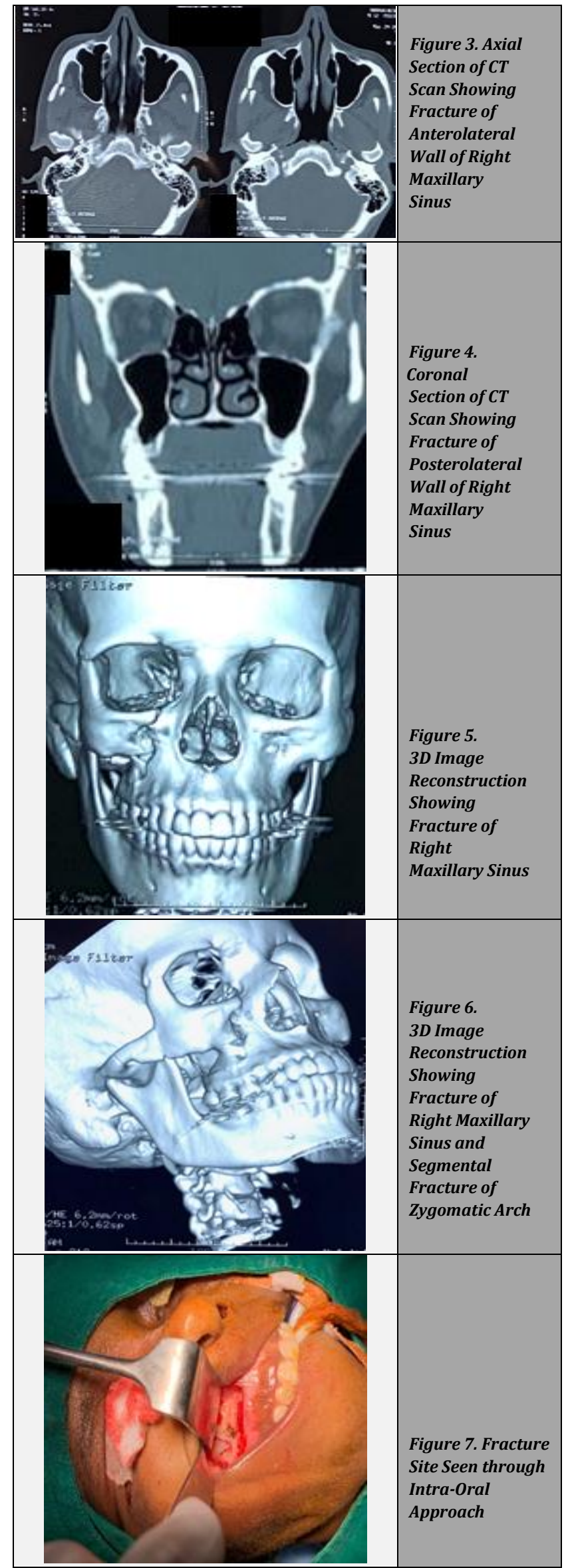




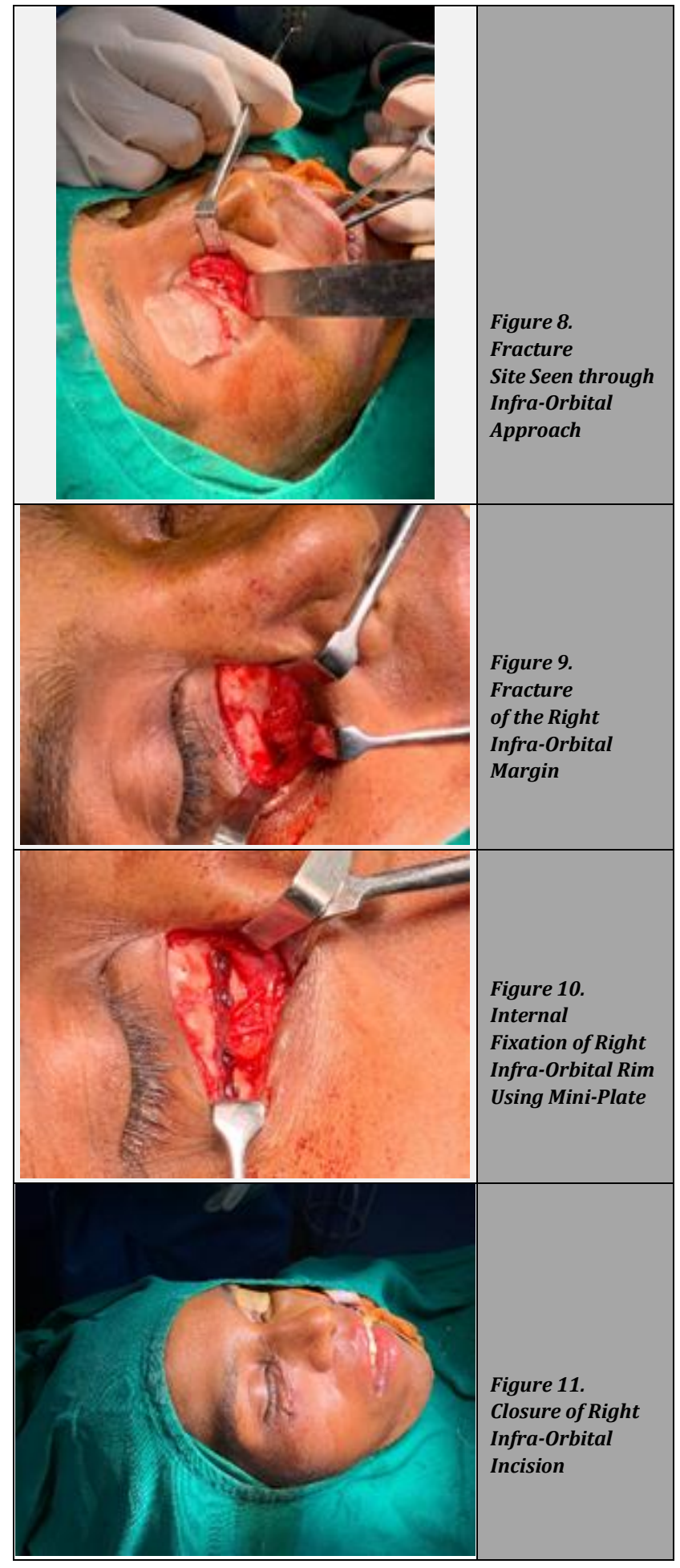

DISCUSSION

Surgical intervention in the form of accurate reduction and fixation is necessary for displaced zygomatic complex fractures. The number of surgical approaches and sites of fixation required to ensure this will vary based on the type of injury and the experience of the operating surgeon.

An intact zygoma (or zygomatic bone) and its surrounding bony anatomy are essential for maintaining facial contour, such as cheek prominence, as well as orbital integrity. ${ }^{5}$ Anatomically, the zygoma is attached to the frontal bone (via the frontozygomatic suture), the maxilla (via the zygomaticomaxillary suture), the squamous part of the temporal bone (via the zygomaticotemporal suture) and the sphenoid bone (via the zygomaticosphenoid suture). Fractures involving zygoma often occur at these four suture sites, leading to a tetrapod fracture pattern, known as a zygomatic complex fracture. ${ }^{6}$

The aetiology of ZMC fractures are influenced by multiple factors, including geographical location, incidence of road traffic accidents and socio-economic trends. Review of literature shows that aetiology is variable, with one study in Poland quoting assault as the most common whereas other studies from Brazil and Amsterdam showing road traffic accidents to be the most common. ${ }^{4,7}$ Fracture patterns may include isolated zygomatic arch fractures or ZMC fractures can be associated with other facial fractures such as nasal bone fractures and Le Fort fractures. ZMC fractures may lead to aesthetic defects such as cheekbone flattening or functional defects which include restrictive mouth opening, malocclusion or ophthalmic issues such as diplopia, restricted eye movements, enophthalmos and hypoglobus. ${ }^{12}$

Over the years, CT scanning has replaced plain radiography as the imaging modality of choice. Almost all zygomatic complex fractures require direct CT scanning in both the axial and coronal planes (less than $3 \mathrm{~mm}$ slice thickness) to categorize the pattern of injury clearly and plan on subsequent management. In the most complex injuries and those involving multiple planes, three-dimensional CT imaging can be used to help the surgeon better visualize fracture fragments. ${ }^{13}$

There are currently no widely accepted treatment protocols or guidelines on the surgical management of ZMC fractures. Open reduction and internal fixation (ORIF) is often the preferred surgical treatment for displaced and comminuted zygomatic complex fractures. The number and location of miniplates for fixation are variable because they depend on the fracture anatomy, extent and amount of displacement. The fixation points commonly used in the open reduction and internal fixation of ZMC fractures include the region of zygomaticomaxillary buttress, frontozygomatic suture and infraorbital margin. ${ }^{12}$ A review of the literature shows that for ORIF of ZMC fractures, the number of fixation points used, their location, as well as the incisional access to these fixation points is variable. ${ }^{2-4}$

A multidisciplinary survey by Farber et al. ${ }^{14}$ in 2016 involving otorhinolaryngology, plastic and oral and maxillofacial surgeons, demonstrated variable treatment choices for ZMC fractures regarding the location and number of fixation points, surgical approaches, as well as the need for orbital floor exploration. With regards to one point fixation, there is variable support from the literature regarding its efficacy, and there is no consensus regarding the optimum anatomical position for one point fixation between the zygomaticomaxillary buttress, the infraorbital margin and the frontozygomatic region, as well as the optimum surgical access to these anatomical fixation points.4,14-16 It is generally accepted that sufficient stability is obtained with one-point fixation when there is no comminution of the ZMC fracture, with two-point and three-point fixation providing increased stability where ever necessary and often based on fracture comminution and surgeon's preference. ${ }^{17}$ The upper buccal sulcus incision through intraoral approach is widely accepted as the best approach for exposure of fracture site in ZMC for 
fixation. The surgeon's experience, training background, and preferences play a significant role in contributing to the variety of surgical approaches to ZMC fractures. ${ }^{12}$

\section{CONCLUSIONS}

Optimal management of displaced zygomatic complex fractures involves proper reduction of fractured segments followed by internal fixation for accurate alignment and stabilization. Early diagnosis and surgical intervention with accurate timing are important in ZMC fractures. Optimal management of displaced zygomatic complex fractures involves proper reduction of fractured segments followed by internal fixation for accurate alignment and stabilization.

Financial or other competing interests: None.

Disclosure forms provided by the authors are available with the full text of this article at jemds.com.

\section{REFERENCES}

[1] Marinho ROM, Freire-Maia B. Management of fractures of the zygomaticomaxillary complex. Oral Maxillofacial Sure Clin North Am 2013;25(4):617-36.

[2] Venugopal MG, Sinha R, Menon PS, et al. Fractures in the maxillofacial region: a four year retrospective study. Med J Armed Forces India 2010;66(1):14-7.

[3] Ribeiro ALR, Da Silva Gillet LC, De Vasconcelos HG, et al. Facial fractures: large epidemiologic survey in Northern Brazil reveals some unique characteristics. J Oral Maxillofac Surg 2016;74(12):2480.e1-12.

[4] Forouzanfar T, Salentijin E, Peng G, et al. A 10 year analysis of the Amsterdam protocol in the treatment of zygomatic complex fractures. J Craniomaxillofac Surg 2013;41(7):616-22.

[5] McBride S, Barry T. Fractures of the zygomatic complex-a comprehensive review over 10 years of surgical management. Br J Oral Maxillofac Surg 2015;53(10):e72.
[6] Dakir A, Muthumani T, Prabu NP, et al. One point fixation of zygomatic tripod fractures in the zygomatic buttress through keen's intraoral approach: a review of 30 cases. J Pharm Bioallied Sci 2015;7(Suppl 1):S238-41.

[7] Bogusiak K, Arkuszewski P. Characteristics and epidemiology of zygomaticomaxillary complex fractures. J Craniofac Surg 2010;21(4):1018-23.

[8] Hwang K, Kim DH. Analysis of zygomatic fractures. J Craniofac Surg 2011;22(4):1416-21.

[9] Farber SJ, Nguyen DC, Skolnick GB, et al. Current management of zygomaticomaxillary complex fractures: a multidisciplinary survey and literature review. Craniomaxillofac Trauma Reconstr 2016;9(4):313-22.

[10] Ellstrom CL, Evans GR. Evidence based medicine: zygoma fractures. Plast Reconstr Surg 2013;132(6):1649-57.

[11] Jensen TS, Linnebjerg LB, Jensen JD. Treatment of zygomatic complex fractures with surgical or nonsurgical intervention: a retrospective study. Open Dent J 2018;12:377-87.

[12] Bradley D, Leung B, Saxena S, et al. Surgical management of zygomatic complex fractures in a major trauma centre. Plast Aesthet Res 2019;6:11.

[13] Hollier LH, Thornton J, Pazmino P, et al. The management of orbitozygomatic fractures. Plast Reconstr Surg 2003;111(7):2386-93.

[14] Farber SJ, Nguyen DC, Skolnick GB, et al. Current management of zygomaticomaxillary complex fractures: a multidisciplinary survey and literature review. Craniomaxillofac Trauma Reconstr 2016;9(4):313-22.

[15] Habal MB. The orbits: it is less important what you put in than how you secure it. J Craniofac Surg 2010;21(4):9656.

[16] Olate S, Lima SM Jr, Sawazaki R, et al. Surgical approaches and fixation patterns in zygomatic complex fractures. J Craniofac Surg 2010;21(4):1213-7.

[17] Baylan JM, Jupiter D, Parker WL, et al. Management of zygomatic fractures: a national survey. J Craniofac Surg 2016;27(6):1571-5. 